\title{
RDF-BASED KNOWLEDGE MODELS FOR NETWORK MANAGEMENT
}

\author{
Jun Shen and Yun Yang
}

Center for Internet Computing and E-Commerce, School of Information Technology, Swinburne University of Technology, PO Box 218, Melbourne, Australia, 3122

\{jshen,yyang\}@it.swin.edu.au

\begin{abstract}
SMIng (next generation structure of management information), an information model for network management, is a prospective structure of management information. When deploying the multi-agent systems to network management environments, we have established a lightweight self-contained knowledge model based on RDF (Resource Description Framework) and its extensions. We also present an implementation prototype to support agent communication and coordination by RDF-based languages.
\end{abstract}

Key words: information modeling, mobile agents, knowledge frameworks

\section{INTRODUCTION}

Mobile and intelligent agents play active roles in network management platforms and products nowadays. Meanwhile, new information models and protocol interfaces are emerging within the Internet communities, for example Script MIB (Management Information Base) [1]. The next generation structure of management information has become critical and the work towards SMIng [2] is in progress.

However, agent communications are critical when deploying multi-agents system to network management platforms. In our previous prototype [3], KQML [4] was used among managing agents by taking advantage of JatLite toolkit (java.stanford.edu). The basic contents of agents' dialogs include script codes and attribute-value pairs, but they lack sufficient support to describe relations and semantics of either managing agents or managed agents.

In this paper, we discuss how to construct a self-contained knowledge model based on the RDF (Resource Description Framework) specifications [5] and their extensions. Our seed information model is SMIng, which is independent of ASN.1 but explicitly defines terms that had been derived from former versions of structure of management information (SMI) [6]. SMIng is devised as a long-term network information model and has a minimal but complete set of data types [3].

The original version of this chapter was revised: The copyright line was incorrect. This has been corrected. The Erratum to this chapter is available at DOI: 10.1007/978-0-387-35674-7_66 
Nevertheless, intelligent management agents should understand each other through a language with more formal semantics.

In comparison to other content languages, the triples of RDF statements in XML syntax describe relations between resources and properties naturally and flexibly. $\mathrm{RDF}$ and RDF Schema (RDFS) have absorbed theories of object-oriented programming, relational databases and knowledge representations and well adapted to semantic Web. The most important enrichment of RDF used by us is OILOntology Inference Layer [7] and its extension and integration with agent language DAML [8], as well as RDF Context [9] and FIPA-RDF [10]. At current stage, similar to Common Information Model (www.dmtf.org), XML has been incorporated to specify DTD or schema of SMIng (www.ibr.cs.tubs.de/projects/sming). Therefore, XML versions of SMIng provide a basic tag vocabulary to link up a more complex management knowledge model.

\section{RDF DESCRIPTIONS OF SMING MODULES}

All object variable resources of the SNMP architecture can be described in the RDF framework. RDFS description is modeling SMIng modules while the RDF model specification modeling SMIng instances, which is actually MIB.

Every SMIng module has its namespace, which is identified by its authors' organisation and its version. We define a namespace xmlns:sming in order to describe meta classes. Besides seven basic data types, other data types are defined as subclasses. The common statements within the SMIng parameter blocks, such as 'default', correspond to properties like rdfs:comment. These properties share the

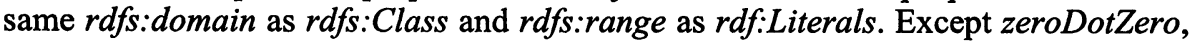
every node may have its corresponding identifier like 'Parent.Key', where 'Parent' is the identifier of its parent node. A 'scalar' statement will be in the following RDFS form - a whole table will be a nested form:

$<$ rdfs:Class ID="ScalarVariableIdentifier">

$<$ rdfs:subClassOf rdf:resource $="$ ParentIdentifier" $>$

$<$ rdfs:subClassOf rdf:resource $=" \#$ DataType" $>$

$</$ rdfs:Class $>$

Instances of SMIng modules are implementations of MIB, which is abstracted as a set of statements that declare values of managed object variables within a specific managing or managed entity at a specific time point. We introduce properties sming:time and sming:value with rdfs:domain as ScalarVariableIdentifier or ColumnIdentifier. The RDF description of instances of column objects will be a nest structure of $r d f: S e q, \quad r d f: l i$ and $r d f: B a g$ of similar instance values of class ScalarVariableIdentifier (xmlns:agent is a reification of $x m \operatorname{lns}: s m i n g$ ):

$<$ agent:ScalarVariableIdentifier $>$

<sming:time>yy:mm:dd:hh:mm:ss</sming:time>

$<$ sming:value $>$ value of certain DataType $</$ sming:value $>$

$<$ agent:ScalarVariableIdentifier $>$

More specification details are discussed in [11].

\section{IMPLEMENTATION OF KNOWLEDGE BASES}

Relational MIB, which is developed based on traditional SMI, may be extended to the management knowledge base with rich semantic capabilities of RDFS. 
Assuming every management agent has a predicate set, rules set and action scripts set, we apply the RDF context and FIPA-RDF to describe agent's knowledge base. sming:value becomes a basic predicate of $\mathrm{MKB}$, while a description of a MIB variable instance becomes a proposition specification of subject-predicate-object and truth-value relationship (fipa:Proposition).

The rich predicates of $\mathrm{MKB}$ may replace sming:value in order to describe more complex relationships between managed resource objects. Similarly, the operations on managed objects are expanded with new management action scripts. Compared with Script MIB, our knowledge base system for network management can provide more diversified functions with more flexibility [11]. Within FIPA-RDF, the rules are regarded as compositions of two parts: selection and manipulation. fipa:selection selects resources according to the specific expressions with the SQL-like RDF Query specification (www.w3.org/TandS/QL/), and fipa-manipulation describes corresponding actions. The management process of every agent is the replicated applications of fipa:Rule or sming:Rule to invoking fipa:Action so as to operate on sets of fipa:Proposition, which are defined by rdfc:asserts or $r d f c$ :assumes within a certain rdfc: Context.

Our prototype is based on MCT (Mobile Code Toolkit) [12] and JMX. KQML messages become carriers of contents of dialogs between agents. The KQML message parameters are redefined, :ontology becomes SMIng modules and : language should be languages of RDF and its extensions. Besides SiRPAC (Simple RDF Parser and Complier), there are also some available toolkit packages for processing the RDF syntax, for example, RDF API (wwwdb.stanford.edu/ melnik/), and Jena (www-uk.hpl.hp.com/people/bwm/rdf/jena/), which may run upon popular XML parsers such as SAX (megginson.com/SAX) as well as Xerces (xml.apache.org/xerces-j/).

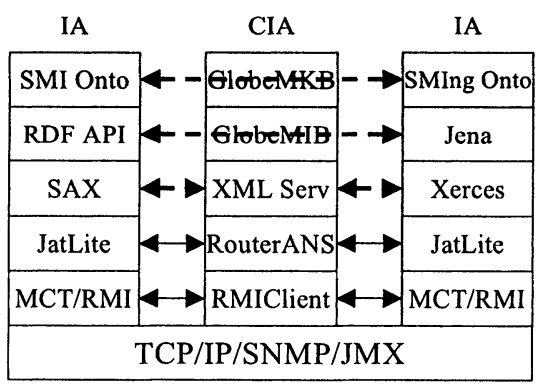

Figure 1. Multi-Agent Implementation Model

The whole implementation model is shown in Figure 1, where Router/ANS helps to route messages. The CIA (coordinating intelligent agent) maintains the global knowledge of a group of intelligent agents (IA), they communicate with each other in SMI or SMIng ontology. The dialogs are encapsulated packets with the headers of $\mathrm{RDF}(\mathrm{S})$ and XML as well as the performatives of KQML. RDF-enabled agents can understand each other by explicit semantics, for example, special requests for coordination and inferences are added-values to traditional network management frameworks, which solely query and manipulate on static variables. 


\section{CONCLUSIONS}

Knowledge representation among management agents is a critical issue. We attempt to establish a lightweight knowledge model based on RDF. With SMIng acting as a seed, mapping from SMIng modules and related MIB (management information base) onto RDF schema (RDFS) definitions of classes, properties and related descriptions has become feasible. Moreover, elements of the management knowledge base, especially, rule bases and action scripts can be described by RDF Context, FIPA-RDF and OIL. Our implementation model integrates Java based tools at different levels to coordinate agents more effectively.

\section{ACKNOWLEDGEMENTS}

This work is partly supported by Swinburne Vice Chancellor's Strategic Research Initiative 2002-2004. The early prototype was implemented at Southeast University and funded by Chinese National Science Foundation (No. 90204009).

\section{REFERENCES}

[1] J. Schönwälder, J. Quittek, C. Kappler, Building distributed management applications with the IETF Script MIB. IEEE Journal on Selected Areas in Communications, Vol.18, No.5, pp.702-714, 2000

[2] C. Elliot, D. Harrington, J. Jason, J. Schönwälder, F. Strauß and W. Weiss, SMIng objectives. IETF Request for Comments 3216, December 2001

[3] J. Shen, J. Luo, G. Gu, An object-oriented net graph model for agent group-based network management. In: Proceedings of Technology of Object-Oriented Language and Systems, (TOOLS 31), IEEE Press, pp.126-132, Sept. 1999

[4] T. Finin, R. Fritzson, D. Mckay, R. McEntire, KQML as an agent communication language. In: Proceedings of the 3rd International Conference on Information and Knowledge Management (CIKM'94), ACM Press, pp.456-463, Nov. 1994

[5] S. Decker, S. Melnik, F. van Harmelen, D. Fensel, M. Klein, J. Broekstra, M. Erdmann, I. Horrocks, 2000, The semantic web: The roles of XML and RDF. IEEE Internet Computing, Vol. 4, No. 5, pp.63-74, 2000

[6]K. McCloghrie, D. Perkins, J. Schoenwaelder, J. Case, M. Rose and S. Waldbusser, Structure of Management Information version 2 (SMIv2). IETF Standard 58, Request For Comments 2578, April 1999

[7] I. Horrocks, et al., The Ontology Inference Layer OIL. Technical Report of On-ToKnowledge Project in the IST Program, http://www.ontoknowledge.org/oil, 2000

[8]D. Connolly, et al., DAML+OIL reference description. World Wide Web Consortium Note, http://www.w3.org/TR/daml+oil-reference, 2001

[9] G. Klyne, Context for RDF information modelling. Available at http:// public.research.mimesweeper.com / RDF / RDFContexts.html, Oct. 10, 2000

[10] FIPA, FIPA Content Language Library. FIPA 99 Specification, v 0.2, Part 18, Foundation for Intelligent Physical Agents, Geneva, Switzerland, 1999

[11] J. Shen, Research on Multi-Agent System Based Network Management Models, Ph.D. Thesis, Southeast University, Nanjing, China, 2001

[12] Bieszczad, Mobile agents for network management, IEEE Communications Surveys, http://www.comsoc.org/pubs/surveys. Fourth Quarter, Vol. 1 No.1, 1998 